\title{
Two-level domain decomposition algorithm for a nonlinear inverse DOT problem
}

\author{
Kiwoon Kwon ${ }^{a}$, Il-young Son ${ }^{a}$, and Birsen Yazici ${ }^{a}$ \\ ${ }^{a}$ Electrical, Computer, and Systems Engineering, Rensselaer Polytechnic Institute
}

\begin{abstract}
Diffuse optical tomography is to find the value of optical coefficients in a tissue using near infra-red lights, which is usually modelled by an optimization problem being composed of two steps: the forward solver to compute the photon density function and the inverse solver to update the coefficients based on the forward solver. Since the resulting problem is mathematically nonlinear ill-posed inverse problem and numerically large-scale computational problem demanding high quality image, it is highly desirable to reduce the amount of computation needed. In this paper, domain decomposition method is adopted to decrease the computation complexity of the problem. Among many methods of domain decomposition techniques, two level multiplicative overlapping domain decomposition method and two level space decomposition method are used to the forward and inverse solver, respectively. The convergence and computational cost of each method are described. And the efficiency of using combined two methods is verified by the implementation of reconstructing the absorption coefficient on square domain and thin domain.
\end{abstract}

\section{INTRODUCTION}

DOT is a nonlinear and ill-posed problem, since biological tissues under examination are turbid and scattering medium. Hence, it is a computationally challenging problem that calls for the use of nonlinear minimization methods with regularization to stabilize the solution.

Based on frequency-domain photon diffusion equation, one of popular method for the forward model, absorption and scattering coefficients are reconstructed to minimize the error between the measured photon density and the computed photon density.

We now present an overview of our problem formation and the methods used in its solution. The overall problem is posed as an optimization problem. It is formulated using two coupled steps, aptly named forward and inverse. Each consists of an iterative solver whose solutions are used as an input to the other solver. More precisely, the forward solver computes the photon density and its Jacobian with respect to the optical coefficients, and the inverse solver updates the optical coefficients based on the output of the forward step. The updated coefficients are then used in the forward solver to recompute the photon density and its Jacobian and so on.

The computational complexity of DOT reconstruction quickly grows with number of pixels and dimensions. Thus, real time computation of DOT needs further numerical techniques to simplify the complexity of the problem. We suggest the use of domain decomposition methods to tackle this problem. Domain decomposition methods have previously been studied in the area of numerical solution to partial differential equations over the last two decades, motivated by the need for fast and efficient algorithm for solving large-scale, three-dimensional problem. One advantage of domain decomposition method is that it allows for distributed parallel numerical solvers on smaller subdomains, making the computation extremely efficient.

In this paper, we will use Algorithm TMODDM (Two-level multiplicative overlapping domain decomposition method) for the forward solver. It is reported that this algorithm approximates photon densities well. For the detailed description and the property of Algorithm TMODDM see. ${ }^{34}$

The main focus of this paper is to apply the idea of two-level Domain decomposition to the inverse solver minimizing optical coefficients. Recently, there is an approach using parallel algorithm 
for unconstrained convex minimization problem. ${ }^{13,14}$ Badea and Tai et al. ${ }^{15-22}$ have previously applied domain decomposition techniques to a constrained convex minimization problem coming from variational inequalities using space decomposition method. It has been shown that multigrid can be viewed as a special case of such space decomposition. ${ }^{23}$ We apply this space decomposition idea to DOT imaging. The present algorithm for inverse solver is as follows. First, we divide the whole domain into disjoint subdomains considering finite elements as in the forward solver. Second, coarse level image of the optical coefficients are computed using data restricted to the coarser grid. Third, using coarse level optical coefficients as initial guess, finer level coefficients are computed at each subdomins. Local convergence of the inverse solver, when the variance of the optical coefficients are small, is proved using the abstract convergence theorem presented in. ${ }^{15}$ It is shown that the two level approach is indispensable in the algorithm due to the properties of local convergence result.

In summary, two domain decomposition technique are used in the present work. One is the twolevel overlapping domain decomposition method (Algorithm TMODDM) for forward solver and the other is two-level space decomposition method (Algorithm TMSDM) for inverse solver. We show that efficiency is gained using these two algorithms with respect to the overall computational complexity of the problem. Absorption coefficient, which is more important in clinical application rather than diffusion coefficient in, ${ }^{34}$ is considered based on more practical assumptions for the parameters involved in the photon diffusion equation.

The paper is organized as follows: in the next section, forward solver based on photon diffusion equation model and minimization formulation of the inverse DOT is described. Two algorithms applying domain decomposition techniques to the forward and inverse steps of the minimization formulation is presented in Section 3. Finally in Section 4, we summarize the results of implementing these algorithms for a simulated problem.

\section{DIFFUSE OPTICAL TOMOGRAPHY}

\subsection{Photon Diffusion Equation in Frequency Domain}

Propagation of light in biological tissues $\Omega$ is described by photon diffusion equation in frequency domain derived from the simplest but non-trivial approximation in spherical harmonics of the Boltzmann equation.

$$
\begin{array}{rl}
-\nabla \cdot(\kappa(r) \nabla \Phi(r, \omega))+\mu_{a} \Phi(r, \omega)+\frac{i \omega}{c} \Phi(r, \omega)=q_{0}(r, \omega) & r \in \Omega \\
\Phi(r, \omega)+2 a \frac{\partial \Phi(r, \omega)}{\partial \nu}=0 & r \in \partial \Omega
\end{array}
$$

where $\Phi$ is the photon density, i.e. an integration of the number of photon per unit sphere, and $\mu_{a}, \mu_{s}^{\prime}, \kappa=\frac{1}{3\left(\mu_{a}+\mu_{s}^{\prime}\right)}$ are the absorption coefficient, the reduced scattering coefficient and the diffusion coefficient, respectively. The constant $a$, is related to the refraction on the boundary and $q_{0}$ is a source term. (2.1b) is called Robin boundary condition, which restricts the inward directed current to zero.

Usual assumption for the source term $q_{0}(r, \omega)=\delta\left(r-r_{0}\right)$ located at some point in $\bar{\Omega}$ is made. Consider $N_{s}$ sources and $N_{d}$ detectors at the boundary of $\Omega$. Let the photon density for each source term $q_{j}(r, \omega)=\delta\left(r-r_{j}\right), j=1, \cdots, N_{s}$ be $\Phi_{j}$. The measured boundary quantity is the normal component of the photon current, defined as the average number of photon in a given direction or simply $\frac{1}{2 a} \Phi_{j}$ due to Robin boundary condition (2.1b). Let detectors be $m_{i}, i=1, \cdots, N_{d}$. Then measured data at the boundary is described by $N_{s} * N_{d}$ column vector $\Phi_{i j}=\log \left(\Phi_{j}\left(m_{i}\right)\right)$ for $i j=$ $j+N_{d} *(i-1)$ for given $\mu_{a}$ and $\mu_{s}^{\prime}$. We have used Rytov approximation for the measurement data which is known to give better image quality than Born approximation as in. ${ }^{24}$ Let measured data at $i$-th detector and $j$-th source be $\Gamma_{i j}$. Diffuse optical tomography is interpreted as finding the coefficients $\mu_{a}$ and/or $\mu_{s}^{\prime}$ in some admissible class to minimize the square error between measured data $\Gamma_{i j}$ and the computed data $\Phi_{i j}\left[\mu_{a}, \mu_{s}^{\prime}\right]$ attained by solving (2.1). 


\subsection{Forward solver using finite element method and adjoint method}

In this section, solution to forward problem is described for computing the boundary data and its Jacobian. Finite element method is used to solve for the photon densities and adjoint method is used to compute the Jacobian.

Consider the finite element space with basis functions $u_{i}^{\prime}, i^{\prime}=1, \cdots, N_{n}$ for some positive number $N_{n}$. Then the finite element formulation for (2.1) in this finite element space for $j$-th source $(j=$ $\left.1, \cdots, N_{s}\right)$ is as follows:

$$
\left[K(\kappa)+C\left(\mu_{a}\right)+1 /(2 a) A+i \omega / c B\right] \Phi_{j}=q_{j}
$$

where

$$
\begin{aligned}
K_{i^{\prime} j^{\prime}} & =\int_{\Omega} \kappa \nabla u_{i}^{\prime} \nabla u_{j}^{\prime} \\
C_{i^{\prime} j^{\prime}} & =\int_{\Omega} \mu_{a} u_{i}^{\prime} u_{j}^{\prime} \\
B_{i^{\prime} j^{\prime}} & =\int_{\Omega} u_{i}^{\prime} u_{j}^{\prime} \\
A_{i^{\prime} j^{\prime}} & =\int_{\partial \Omega} u_{i}^{\prime} u_{j}^{\prime}
\end{aligned}
$$

for $N_{n} \times N_{n}$ matrices $K, C, B, A$ and indices $i^{\prime}, j^{\prime}=1, \cdots, N_{n}$. By restricting $\Phi_{j}$ on detectors $m_{i}, i=$ $1, \cdots, N_{d}$, we obtain the boundary measurement data $\Gamma_{i j}$.

To implement the minimization procedure for optical coefficients, aside from computing $\Gamma_{i j}$ obtained from (2.2), the derivative of $\Gamma_{i j}$ with respect to the optical coefficients is needed. If we use Newton quotient for the computation of the derivative, computational burden increases proportional to the number of dimension of the space of optical coefficients. To reduce the computational burden, Jacobian is computed by the adjoint method using Rytov approximation or Born approximation. The assumption for both Rytov and Born approximation is the variance of optical coefficients and related photon densities are small as in. ${ }^{24}$ This smallness assumption will be important factor in Section 3.2. Suppose piecewise linear finite element $P_{1}$ or piecewise bilinear finite element $Q_{1}$ is used. Suppose further that the diffusion and absorption coefficients are constant in each finite element. Let $N_{e}$ be the number of finite element in $\Omega$. The derivative of $\Gamma_{i j}$ is different depending on which optical parameters system we choose. For parameter system $\left(\kappa, \mu_{a}\right)$, let the derivatives of $\Gamma_{i j}$ with respect to $\kappa$ and $\mu_{a}$ be $\widetilde{J}^{\kappa}$ and $\widetilde{J}^{\mu_{a}}$. Whereas for parameter system $\left(\mu_{a}, \mu_{s}^{\prime}\right)$, let the derivatives be $J^{\mu_{s}^{\prime}}$ and $J^{\mu_{a}}$. Then for $j$-th source, $i$-th detector, and $k$-th element, each four derivatives, $(N s * N d) \times N e$ matrices, are computed by

$$
\begin{aligned}
\widetilde{J}^{\kappa}(i j, k) & =1 /(2 a) \frac{\sum_{l, m=1}^{N n} \Phi_{l, i}^{*} V_{k}(l, m) \Phi_{m, j}}{\Phi_{i, j}} \\
\widetilde{J}^{\mu_{a}}(i j, k) & =1 /(2 a) \frac{\sum_{l, m=1}^{N n} \Phi_{l, i}^{*} W_{k}(l, m) \Phi_{m, j}}{\Phi_{i, j}} \\
J^{\mu_{s}^{\prime}} & =\widetilde{J}^{\kappa} \frac{\partial \kappa}{\partial \mu_{s}^{\prime}}=-3 \kappa^{2} \widetilde{J}^{\kappa} \\
J^{\mu_{a}} & =\widetilde{J}^{\kappa} \frac{\partial \kappa}{\partial \mu_{a}}+\widetilde{J}^{\mu_{a}}=-3 \kappa^{2} \widetilde{J}^{\kappa}+\widetilde{J}^{\mu_{a}}
\end{aligned}
$$

where

$$
\begin{aligned}
V_{k}(l, m) & =\int_{T_{k}} \nabla u_{l} \nabla u_{m} \\
W_{k}(l, m) & =\int_{T_{k}} u_{l} u_{m}
\end{aligned}
$$


for $k$-th element $T_{k}$. Since optical parameters $\left(\mu_{a}, \mu_{s}^{\prime}\right)$ are more important optical parameters, we will use these parameter system from now on in this paper.

\subsection{DOT as a nonlinear ill-posed optimization problem}

As stated in the introduction, DOT is a nonlinear ill-posed problem. Thus, regularization is needed to constrain and stabilize the solution. We chose Tikhonov type regularization. Newton-type, trust region method is used, which gives good results for large-scale constrained optimization problem of the type considered in this paper. Newton-type method is used to consider Hessian of the object value of the nonlinear optimization. Among various Newton-type method, trust region method is used in this paper, which is good for large-scale constrained optimization problem.

Given forward solver computing photon densities $\Gamma_{i j}$ at the boundary $\partial \Omega$ ( and its Jacobian $J_{i j, k}$ ), optical coefficients are estimated by the following inverse problem minimizing the error between measured boundary data and solution of the forward solver. Let $V$ be some admissible space of optical coefficients $\kappa$ and $\mu_{a}$.

$$
\min _{\kappa, \mu_{a} \in V} F\left(\kappa, \mu_{a}\right), \quad F\left(\kappa, \mu_{a}\right)=\frac{1}{2} \sum_{j=1}^{N_{s}} \sum_{i=1}^{N_{d}}\left(\frac{\Phi_{i, j}\left(\kappa, \mu_{a}\right)-\Gamma_{i, j}}{\sigma}\right)^{2}+\alpha \Upsilon\left(\kappa, \mu_{a}\right),
$$

where $\sigma$ is a standard deviation, $\alpha$ is the regularization parameter, and $\Upsilon$ is some measure in $V$. For the computational convenience assume $\sigma=1$ for the rest of this paper.

The unique identification of $\kappa$ and $\mu_{a}$ in (2.1) for infinite sources and infinite measurements when $\omega \neq 0$ can be easily shown by using the uniqueness results for isotropic case ${ }^{25}$ or for the case having anisotropic anomalies. ${ }^{26-28}$ But the uniqueness of $\kappa, \mu_{a}$ is not known generally for the finite sources and detectors. Thus the unique solvability of (2.4) is not assured leading to the ill-posedness of the DOT problem. But local uniqueness and convergence of (2.4) having positive Tikhonov regularization parameter is attained using local strong convexity of the operator $F$, which will be shown in (3.3).

The method of solving (2.4) is composed of two steps; the step to find the minimizing directions $\delta \kappa$ and $\delta \mu_{a}$ at the current coefficients $\kappa$ and $\mu_{a}$ and the step to perform line search on those minimizing directions. In the Newton-type method, minimizing direction is $-\left(F^{\prime \prime}\right)^{-1} F^{\prime}$, where $F^{\prime}$ and $F^{\prime \prime}$ are gradient and Hessian of $F$ with respect to $\kappa$ and $\mu_{a}$. In the classical Newton method, searching direction is found by computing $F^{\prime \prime}$ directly. But this method needs heavy computation and is unstable. To decrease complexity of the computation, in quasi-Newton method, $F^{\prime \prime-1}$ is approximated by various cheaper methods such as Davidon-Fletcher-Powell method, Broyden-Fletcher-GoldfarbShanno method, and conjugate gradient method. ${ }^{29}$ To stabilize the computation, $F^{\prime \prime}+\lambda I$ is used in place of $F^{\prime \prime}$ in Levenberg-Marquadt method. ${ }^{30,31}$ Another variance of Newton method is trust region method which One of efficient Newton-type method is a trust region method which computes the following minimization problem

$$
\min \left\{s \in W,\|D s\| \leq \Delta \mid \frac{1}{2} s^{t} F^{\prime \prime} s+s^{t} F^{\prime}\right\}
$$

where $D$ is a scaling matrix, $\Delta$ is a trust region parameter, and $W$ is a subspace of $V$. The role of trust region parameter $\Delta$ is similar to $\lambda$ in Levenberg-Marquadt method. To avoid heavy computation, two dimensional subspace composed of gradient direction and approximate Newton direction is chosen for subspace $W .{ }^{32}$ This trust region method is good for large-scale optimization method as (2.4) having upper bound and lower bound. Thus we will use trust region method as a optimization solver in this paper as in optimization toolbox 3.0 in Matlab. ${ }^{33}$

\section{DOMAIN DECOMPOSITION TECHNIQUES FOR DIFFUSE OPTICAL TOMOGRAPHY}

In this section, we describe the two domain decomposition methods considered in this paper, as applied to forward and inverse solutions. 


\subsection{Two level multiplicative overlapping domain decomposition method}

In forward solver of DOT formulation, we must find photon densities at detectors for all sources and compute its Jacobian with respect to the optical coefficients. Using adjoint method addressed in the previous section, computing Jacobian is straightforward by (2.3) given that photon densities for all sources and for all detectors (assuming detectors as virtual sources) are computed. A commonly used method for computing photon densities is via finite element method as in (2.2). In addition, we apply domain decomposition method to (2.2) in order to reduce the computation complexity of the problem.

The shape of photon density has a peak around the source and decrease rapidly far from the source. Thus one level multiplicative Schwarz method is not sufficient, since the domain far from the sources may neglect the existence of the sources, especially in the case where there are multiple subdomains. Hence we suggest two-level multiplicative overlapping domain decomposition method with following algorithm:

\section{Algorithm TMODDM}

1. Initialization Define subdomains, overlapping regions, maximum number of iterations.

2. Coarse grid correction Implement coarse grid correction using restriction and interpolation operators.

3. Subdomain correction Update photon densities for all sources at each subdomains. To use adjoint method, also compute photon densities for all detectors assuming detector as virtual source.

4. Stopping condition Iterate Coarse grid correction and Subdomain correction until maximum number of iteration is reached or the difference between the newly updated and previous photon densities in the overlapping region is sufficiently small.

5. Boundary measurement data Compute $\Gamma$ by restricting photon densities to the detectors on the boundary

6. Jacobian Compute Jacobian $J$ using adjoint method (2.3).

Suppose that $\Omega$ is a square in $\mathbb{R}^{n}, n=2,3$ discretized with $N^{n}$ mesh. Using a banded direct solver requires $O\left(N^{2 n}\right)$ floating points operations. But if two-level Schwarz method with $d$ decomposition of the domain is used, then the complexity is reduced to $O\left(\frac{N^{2 n}}{d}+N^{n}+d^{2}\right)$. If we use parallel computation with $d$ computers, each computer needs less than $O\left(\frac{N^{2 n}}{d^{2}}+N^{n}+d^{2}\right)$. Note that the number of iteration $M$ is bounded independent of $N^{n}, d$ and $N^{n} / d$ by the above result. Assuming $d<<N^{n}$, we can get approximately $\frac{M}{d}$ times $O\left(N^{2 n}\right)$ complexity for the present method in serial computing, and $\frac{M}{d^{2 n}}$ times $O\left(N^{2 n}\right)$ for the present method in parallel computing without considering data communication. By using multiple subdomains for the present method we achieve significant decrease in computational costs.

\subsection{Two level multiplicative space decomposition method}

We now describe the algorithm used in the inverse solver in detail. Assume $\kappa$ and $\mu_{a}$ are piecewise constant in each elements and let $\kappa$ and $\mu_{a}$ be some constant $\kappa_{l}$ and $\left(\mu_{a}\right)_{l}$ at $l$-th element. Take $V=\left\{x=\left(\kappa, \mu_{a}\right) \mid L \leq \kappa_{l},\left(\mu_{a}\right)_{l} \leq U\right\}$ and $\|x\|_{V}=\sqrt{\sum_{l=1}^{N e} \kappa_{l}^{2}+\left(\mu_{a}\right)_{l}^{2}}$ and $\Upsilon(x)=\frac{1}{2}\|x\|_{V}^{2}$. Then by simple computation,

$$
\Upsilon^{\prime}(x)=x, \quad \Upsilon^{\prime \prime}(x)=I_{2 N e} .
$$

where $I_{2 N e}$ is $2 N e \times 2 N e$ identity matrix. 
Let $b=\Phi\left(\kappa, \mu_{a}\right)-\Gamma$ and the Hessian of $b$ with respect to $\kappa, \mu_{a}$ be $H$, then $F^{\prime}$ and $F^{\prime \prime}$ are computed as follows:

$$
F^{\prime}(x)=J(x)^{t} b(x)+\alpha x, \quad F^{\prime \prime}(x)=J(x)^{t} J(x)+H(x) b(x)+\alpha I_{2 N e}
$$

Note that since Jacobian $J$, which is independent of $\delta x$ in (2.3) by Born approximation, is given the Hessian $H$ vanishes. Thus we get

$$
\left(\sigma_{2}+\alpha\right)\|y\|_{V}^{2} \leq y^{t} F^{\prime \prime}(x) y \leq\left(\sigma_{1}+\alpha\right)\|y\|_{V}^{2} \text { for } x, y \in V \text { and } x \text { is small. }
$$

for largest singular value $\sigma_{1}$ and smallest singular value $\sigma_{2}$ of $J$. If $N s * N d<2 N e, \sigma_{2}=0$. Even in the case that $N s * N d \geq 2 N e$ it is not clear that $\sigma_{2}>0$. However, with the aid of Tikhonov parameter $\alpha>0$, we can get strong convexity of $F$, from which follows that $C^{\prime}\|x\|_{V}^{2} \leq x^{t} F^{\prime \prime}(x) x$ for positive number $C^{\prime}$.

Convergence analysis for the space decomposition technique is based mostly on the strong convexity of objective function $F$. Which means that since in our case we derived the strong convexity result (3.3) from Born approximation, the convergence hold only if there is but a small variance in the optical coefficients $x$. Thus we need some modification on the previous approach to space decomposition technique. We need a good initial guess for the optical coefficients to be minimized. Incited by Algorithm TMODDM, coarse level optimized optical coefficients is used as an initial guess. As far as the authors' knowledge, how close these initial coarse level solutions are to the true finer level coefficients is not known. For the present work, we have assumed that this is sufficiently close to guarantee local convergence.

Let $\Omega$ be the sum of nonoverlapping $d$ subdomains $\Omega_{m}, m=1, \cdots, d$ and the number of finite elements in each subdomain $\Omega_{m}$ be $N e^{m}$. Then it is obvious that $N e=\sum_{m=1}^{d} N e^{d}$. The coarse level approximation of the coefficients are computed using the boundary data $\Gamma_{i j}^{c}$ on a coarse grid by using restriction operator to the measured data $\Gamma_{i j}$ used in Algorithm TMODDM.

\section{Algorithm TMSDM}

1. Initialization Choose subdomains, maximum number of iteration.

2. Restriction Restrict $\Gamma_{i j}$ to coarse level $\Omega$. Let $\Gamma_{i j}^{c}$ be this restriction boundary data.

3. Coarse level correction Find coefficients $x^{c}$ at this coarse level from $\Gamma_{i j}^{c}$ using Algorithm TMODDM.

4. Upsampling Compute upsampled data $x$ from $x^{c}$ at coarse level.

5. Subspace correction Using $x$ as an initial guess, update at all subdomains sequentially or parallel also using Algorithm TMODDM.

6. Downsampling If maximum number of iteration is reached or stopping condition is satisfied, stop. Otherwise, downsample $x$ to $x^{c}$ to coarse level and go to $\mathbf{3}$ using $x^{c}$ as an initial guess.

Roughly speaking, the computational advantage of space decomposition is as follows. Assume we use Newton direction for minimizing direction of (2.4) at each iterative step. Then we must compute $h$ satisfying

$$
\left(J^{t} J+\alpha I_{2 N e}\right) h=J^{t} b .
$$

(3.4) is solved by preconditioned conjugate gradient method and preconditioner is chosen by considering the structure of $J$. Assuming this preconditioned matrix computation is of order $O\left(N e^{2}\right)$ similar to band solver without space decomposition, the total computation for Algorithm TMSDM for $(3.4)$ is $O\left(d *\left(\frac{N e}{d}\right)^{2}+\left(\frac{N e}{2}\right)^{2}\right)=\left(\frac{1}{d^{3}}+\frac{1}{16}\right) O\left(N e^{4}\right)$ for serial computing and $\left(\frac{1}{d^{4}}+\frac{1}{16}\right) O\left(N e^{4}\right)$ for 
parallel computing. Thus the numerical efficiency is greatly increased for small increase in number of subdomains.

The local linear convergence of Algorithm TMSDM is shown below using Theorem 3.1. in. ${ }^{15}$ Theorem 1. Let $\kappa^{n}$ and $\mu_{a}^{n}$ are $n$-th step approximation for the Algorithm TMSDM and assume that $\kappa^{m+1}-\kappa^{m}$ and $\mu_{a}^{m+1}-\mu_{a}^{m}$ are sufficiently small for all $m=0, \cdots, n-1$. Then we have

$$
\left\|\left(\kappa^{n}, \mu_{a}^{n}\right)-\left(\kappa, \mu_{a}\right)\right\|_{V} \leq C_{1}\left(C_{2}\right)^{n}\left|F\left(\kappa^{0}, \mu_{a}^{0}\right)-F\left(\kappa, \mu_{a}\right)\right|
$$

where $C_{1}$ is a positive constant and $C_{2}$ is a positive constant less than 1 .

Proof. Let $V=V_{1}+\cdots+V_{d}$, where $V_{l}, l=1, \cdots, d$ is the restriction of $V$ into $\Omega_{l}, l=1, \cdots, d$. Then, considering that $V$ is a convex closed subspace of reflexive Banach space $L^{2}(\Omega)$, we need only to show that assumptions $(3.1),(3,2)$ and $(3,3)$ for Theorem $3.1 \mathrm{in},{ }^{15}$ holds for our case to prove the above theorem. These assumption can easily be shown using the local strong convexity convexity (3.3), with the aid of Taylor expansion of $F^{\prime}$, mutual disjointness of $V_{l}, l=1, \cdots, d$, and Cauchy-Schwarz inequality.

\section{NUMERICAL TEST}

For the efficiency of Algorithm TMODDM approximating photon densities, compared to one-level MODDM (Multiplicative Overlapping Domain Decomposition Method), see. ${ }^{34}$ In this section, we will test proposed Algorithm TMSDM with Algorithm TMODDM as its forward solver using simulated data.

In this section, Algorithm TMSDM is implemented under the various kind of $\Omega, \mu_{a}$, sources and detectors location, and Tikhonov regularization parameter. The followings are the coefficients we have chosen for the implementation.

$$
\begin{aligned}
\mu_{s}^{\prime} & =8 \mathrm{~cm}^{-1} \\
\mu_{a} \text { at background } & =0.05 \mathrm{~cm}^{-1} \\
\mu_{a} \text { at tumor } & =0.2 \mathrm{~cm}^{-1} \\
\omega & =2 \pi * 10^{8} \mathrm{~Hz} \\
c & =3 * 10^{10} \mathrm{~cm} / \mathrm{sec} \\
a \text { (reflection coefficient) } & =1
\end{aligned}
$$

The values presented here has more practical meaning than the authors' previous implementation in. ${ }^{34}$ The maximum number of iteration for coarse level and subspace correction for each subdomains are 25 and 10. Coarse level correction is used as an initial guess. Let us denote one cycle for subspace correction for all subdomains, sequentially or parallel. If we use multiple cycle, we can get a better result. But in Figure 1 and 2, we have used just one cycle. As a forward solver we have used Algorithm TMODDM with maximum iteration 3 and overlapping width 1 . The decomposition of subdomains was chosen the same for the forward solver and the inverse solver.

In Figure 1, we have considered $\Omega=[0,1] \times[0,1]$ divided into $20 \times 20=400$ pixels. 20 sources and 20 detectors are located on the boundary as in Figure 1(b). In Figure 1(a), the original image of $1-\mu_{a}$ is depicted. From now on in this paper, $1-\mu_{a}$ is presented to make the background tissue white. The image is reconstructed using $2 \times 2$ subdomains after one cycle. Without regularization, the reconstructed image is disconnected in Fig 1(c), whereas the image Fig 1(d) obtained from Tikhonov regularization is connected but the image quality is not so good.

In Figure 2 , we have considered thin slab type $\Omega=[0,1] \times[0,0.2]$ divided into $20 \times 4$ pixels. We have used 10 sources, 10 detectors as in Figure 2(b). The reconstructed image of absorption coefficient with Algorithm TMSDM with $3 \times 1$ subdomains is presented in Figure 2(c) and Figure 2(d) for Tikhonov parameter 0 and $1 . e-8$, respectively. Compared to Figure 1, we have clearer image. 


\section{ACKNOWLEDGEMENT}

Various portions of this research were supported by the Center for Subsurface Sensing and Imaging Systems, under the Engineering Research Centers Program of the National Science Foundation (Award Number EEC-9986821), and Rensselaer Polytechnic Institute. This material is also based upon work supported by NSF-BES-0353160, ONR-N00014-04-1-0694, and US Army Medical ResearchW81XWH-04-1-0559. Any opinions, findings, and conclusions or recommendations expressed in this material are those of the authors and do not necessarily reflect the views of the National Science Foundation.

\section{REFERENCES}

1. D. A. Boas, D. H. Brooks, E. L. Miller, C. A. DiMarzio, M. Kilmer, R. J. Gaudette, and Q. Zhang, "Imaging the body with diffuse optical tomography," IEEE signal processing magazine, pp. 57$75,2001$.

2. X. Intes, C. Maloux, M. Guven, B. Yazici, and B. Chance, "Diffuse optical tomography with physiological and spatial a priori constraints," Phys. Med. Biol. 49, pp. N155-63, 2004.

3. M. Guven, B. Yazici, X. Intes, C. Maloux, and B. Chance, "Diffuse optical tomography with a priori anatomical information." submitted.

4. M. Dryja, "An additive Schwarz algorithm for two- and three- dimensional finite element elliptic problems," in Domain decomposition methods, pp. 168-72, SIAM, 1989.

5. M. Dryja and O. Widlund, "Towards a unified theory of domain decomposition algorithms for elliptic problems," in Iterative methods for large systems, Academic press, 1990.

6. J. Bramble, J. Pasciak, J. Wang, and J. Xu, "Convergence estimates for product iterative methods with applications to domain decomposition," Math. Comp. 57, pp. 1-21, 1991.

7. P. L. Lions, "On the Schwarz alternating method I," in Proceedings of the first international symposium on domain decomposition methods for partial differential equations, pp. 2-42, SIAM, 1988.

8. P. L. Lions, "On the Schwarz alternating method II," in Domain decomposition methods, pp. 4770, SIAM, 1989.

9. P. L. Lions, "On the Schwarz alternating method III," in Proceedings of the third international symposium on domain decomposition methods for partial differential equations, pp. 202-23, SIAM, 1990.

10. I.-Y. Son, M. Guven, B. Yazici, and X. Intes, "A 2-level domain decomposition algorithm for inverse diffuse optical tomography," in Proceedings of ICIP, IEEE, 2004.

11. B. Smith, P. Bjorstad, and W. Gropp, Domain Decomposition, Cambridge University Press, 1996.

12. A. Quarteroni and A. Valli, Domain Decomposition Methods for Partial Differential Equations, Oxford Science Publications, 1999.

13. D. Conforti and R. Musmanno, "Parallel algorithm for unconstrained optimization based on decomposition techniques," Journal of optimizatin theory and applications 95, pp. 531-44, 1997.

14. O. L. Mangasarian, "Parallel gradient distribution in unconstained optimization," SIAM J. Control and Optimization 33, pp. 1916-25, 1995.

15. L. Badea, X.-C. Tai, and J. Wang, "Convergence rate analysis of a multiplicative Schwarz method for variational inequalities," SIAM J. Numer. Anal. 41, pp. 1052-73, 2003.

16. X.-C. Tai and P. Tseng, "Convergence rate analysis of an asynchronous space decomposition method for convex minimization," Mathematics of Computation 71, pp. 1105-35, 2001.

17. L. Badea and J. Wang, "An additive Schwarz method for variational inequalities," Mathematics of Computation 69, pp. 1341-54, 1999.

18. L. Badea, "On the Schwarz alternation method with more than two subdomains for nonlinear monotone problems," SIAM J. Numer. Anal. 28, pp. 179-204, 1991.

19. X.-C. Tai, "Rate of convergence for some constraint decomposition methods for nonlinear variational inequalities," Numer. Math. 93, pp. 755-86, 2003. 
20. X.-C. Tai, "Some new domain decomposition and multigrid methods for variational inequalities," in Fourteenth International Conference on Domain Decomposition Methods, pp. 323-30, 2003 DDM.org, 2003.

21. J.-P. Zeng and S. Z. Zhou, "A domain decomposition method for a kind of optimization problems," Journal of Computational and Applied Mathematics 146, pp. 127-39, 2002.

22. X.-C. Tai, J. Fr $\phi$ en, M. S. Espedal, and T. F. Chan, "Overlapping domain decomposition and multigrid methods for inverse problems," Contemporary Mathematics 218, pp. 523-29, 1998.

23. J. Xu, "Iterative methods by space decomposition and subspace correction," SIAM Reviews 34, pp. 581-613, 1992.

24. S. R. Arridge, "Optical tomography in medical imaging," Inverse Problems 15, pp. R41-93, 1999.

25. J. Sylvester and G. Uhlmann, "A global uniqueness theorem for an inverse boundary value problem," Ann. math. 125, pp. 153-69, 1987.

26. K. Kwon and D. Sheen, "Anisotropic inverse conductivity and scattering problems," Inverse Problems 18, pp. 745-56, 2002.

27. K. Kwon, "Identification of anisotropic anomalous region in inverse problems," Inverse Problems 20, pp. 1117-36, 2004.

28. V. Isakov, "On uniqueness in the inverse transmission scattering problem," Commun. Partial Diff. Eq. 15, pp. 1565-87, 1990.

29. M. S. Bazaraa, H. D. Sherali, and C. M. Shetty, Nonlinear programming, theory and algorithms, John Wiley \& Sons, Inc., second ed., 1993.

30. K. Levenberg, "A method of the solution of certain problems in least squares," Quart. Appl. Math. 2, pp. 164-68, 1944.

31. D. Marquadt, "An algorithm for least-squares estimation of nonlinear parameters," IEEE Transactions of Circuits and Systems CAS-26,Sept., 1979.

32. R. H. Byrd, R. B. Schnabel, and G. A. Shultz, "Approximate solution of the trust region problem by minimization over two-dimensional subspaces," Mathematical programming 40, pp. 247-63, 1988.

33. Optimization toolbox, for use with matlab, version 3, The Mathworks Inc., 2004.

34. K. Kwon, I.-Y. Son, and B. Yazici, "Domain decomposition for diffuse optical tomography," in Computational Imaging III, C. A. Bouman and E. L. Miller, eds., Proceedings of SPIE 5674, 2005. 


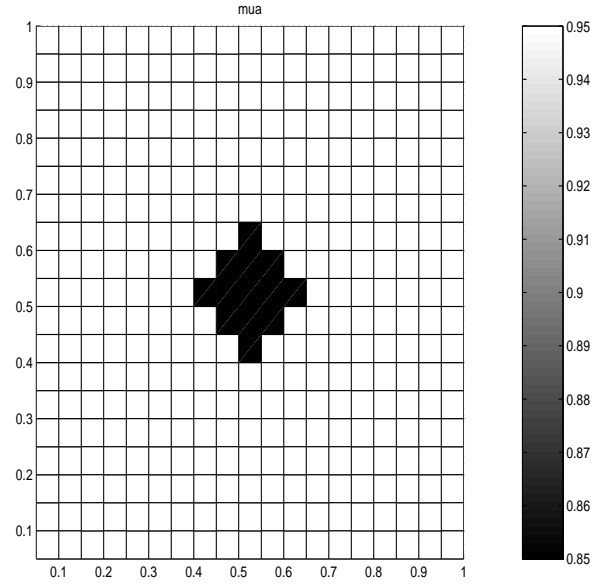

(a)Image of $1-\mu_{a}$ to be reconstructed. White region represent background tissue and black region

represent anomaly which have 4 times larger absorption coefficient than the background.

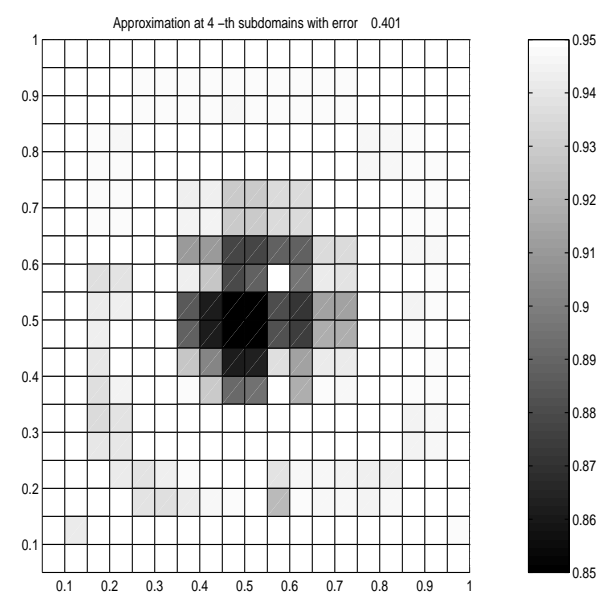

(c)Reconstruction of absorption coefficient with no Tikhonov regularization

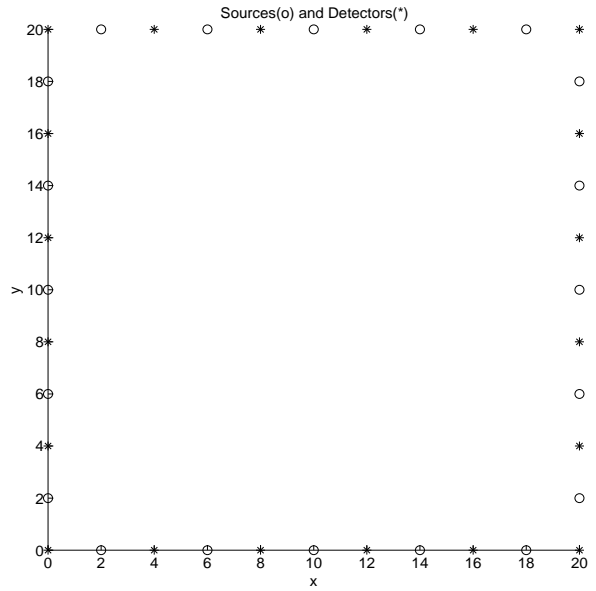

(b)The locations of 20 sources and 20 detectors.

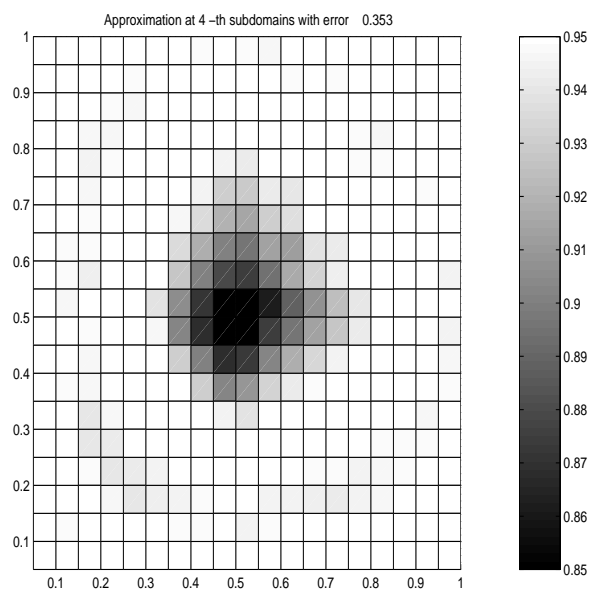

(d)Reconstruction of absorption coefficient with Tikhonov parameter 1.e-7.

Figure 1. Reconstruction of absorption coefficient using Algorithm TMSDM on [0,1] $\times[0,1]$ with $2 \times 2$ subdomains, 20 detectors, 20 sources, and 400 pixels. 


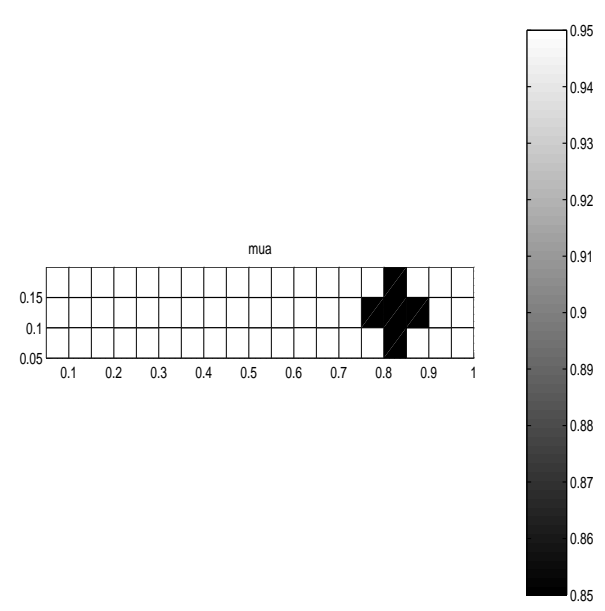

(a)Image of $1-\mu_{a}$ to be reconstructed. White region represent background tissue and black region

represent anomaly which have 4 times larger absorption coefficient than the background.

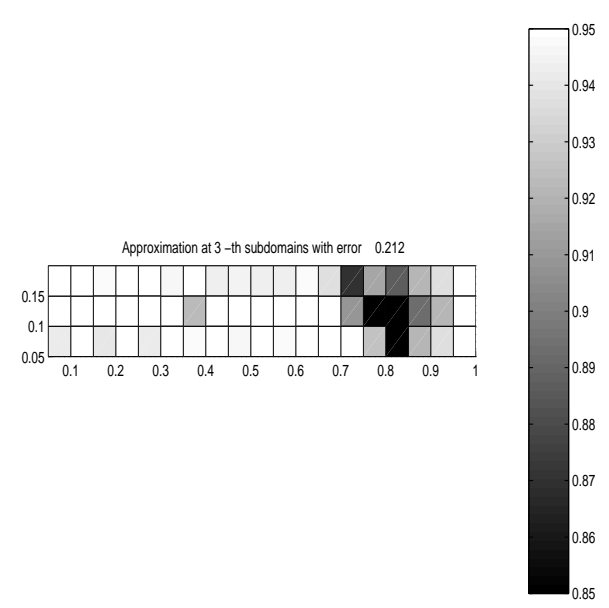

(c)Reconstruction of absorption coefficient with no Tikhonov regularization.

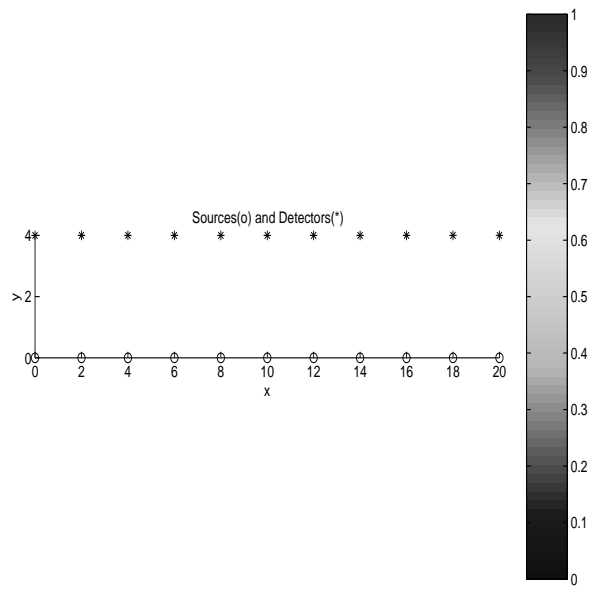

(b)The locations of 10 sources and 10 detectors. 